\title{
OBSERVATIONS OF FAINT STARS DEEP TO 16TH MAGNITUDE WITH CCD MERIDIAN CIRCLE
}

\author{
M. Yoshizawa, S. Suzuki, T. Kuwabara, and H. Ishizaki \\ National Astronomical Observatory \\ Mitaka, Tokyo 181 \\ Japan
}

\begin{abstract}
The experimental works for the development of the CCD micrometer to be used in global astrometry are coming to the final stage at the National Astronomical Observatory, Mitaka. Our goal is to observe and determine the precise optical positions of faint celestial objects deep to 16 th mag with meridian circle. The so-called drift scanning method is adopted as the basic electrical architecture to detect and accumulate the photons from the target objects. Reported in this paper are the performances and some results of the test observations made by using the CCD micrometer equipped to Gautier meridian circle at Mitaka. The present CCD meridian circle was used in 1991 to determine directly the optical position of $3 \mathrm{C} 273 \mathrm{~B}$ in the FK5 system. Also reported are the test observations of the Seyfert galaxy NGC 1068.
\end{abstract}

\section{INTRODUCTION}

With the introduction of the photoelectric meridian circles, it became possible in the late '60s to observe the 12 th mag stars directly with meridian circle. During the last ten years the world four photoelectric meridian circles (at Bordeaux, La Palma, Tokyo, and New Zealand) spent unnegligible fractions of their times to observe the faint reference stars of magnitude between 10 to 12 . The accumulation of those data will lead in near future the construction of reliable faint extension of the present fundamental reference stars.

Now it is natural for the people working with the meridian circles to plan to observe the fainter objects, say deep to 15 th to 17 th mag. Those faint objects (some stellar and others non-stellar) are very important in order to connect the optical (or dynamical) and radio reference frames to each other, and also for the studies of the galactic kinematics. The use of an area CCD is of great advantage to the observations of an overwhelming number of such faint objects, for it is possible with CCD to observe hundreds of stars at once.

The experimental work of developing a CCD micrometer to be used in the global optical astrometry with meridian circles was started at the National Astronomical Observatory, Mitaka, in 1989. The work is now running round the final corner. Our goal is to construct the CCD micrometer that can observe faint celestial objects deep to 16th mag with enoughly high $\mathrm{S} / \mathrm{N}$ ratios. The so-called drift-scanning method (, or SCAN + COMBINED mode according to Monet 1988) is the fundamental architecture in driving CCD of which basic idea 
is described in the next section. The photon statistics of our CCD micrometer are given in section 3 , while in section 4 are presented the results of some test observations made by using the CCD micrometer equipped to Gautier meridian circle at Mitaka.

\section{DRIFT-SCANNING CCD MICROMETER}

In the focal plane of a meridian circle the focused images of celestial objects move regularly with a speed which depends on the declination of each object. In order to follow the diurnal motion of the images without using any mechanical drives the drift-scanning method is used. In the drift-scanning-CCD-drive the photoelectrons stored in a bucket of each CCD pixel are transferred to the next bucket at the timings synchronized to the movement of the diurnal motion by using the declination dependent CCD-drive clock. Thus the geometrical relation between the intensity distribution of the star images and the distribution of the stored electric charges is kept fixed during the course of the drift-scanning. A precise time mark of an atomic clock is attached to each line of data when they are read and fed to A/D converter.

We have constructed an experiment model of the drift-scanning CCD micrometer "DISC" (DIgital Strip scanning Ccd micrometer), which consists of a single-field CCD image sensor TH7883 (Thomson-CSF, France) cooled by liquid nitrogen down to around 170K, a CCDdrive board, 16 bit A/D converter, and EWS (Engineering Work Station) to control the whole system. It was found from the observations of a few open star clusters with good photometric standards that we can reach down to 14.5 mag stars with DISC equipped to Gautier meridian circle at Mitaka. This magnitude limit is a little bit brighter than that of USNO(Flagstaff) CCD transit telescope (cf. Stone 1991), mainly because of the rather bright night sky at Mitaka, and also of large read-out noise of our present system. The performance of DISC is improved by reducing the read-out noises. We expect that the stars of 15 th V-mag can be observed with good S/N ratio by using the improved DISC.

Meanwhile the construction of a new CCD micrometer (hereafter called DISC-II) by using a larger CCD chip, CCD05-30(EEV, U.K.) (1242(H) times 1152(V) pixels of 22.5 $\mu m$ square), and 18 bit A/D converter has been almost finished. DISC-II is to be used for the observations of a part of the second Tokyo PMC Program (1993-) (see section 5). Equipped to the Tokyo PMC one pixel width of DISC-II corresponds to 1.8 arcsec. Thus the frequency of the line drive clock synchronized to the diurnal motion is about $8.3 \cos \delta \mathrm{Hz}$. When cooled down to $140 \mathrm{~K}$, the preliminary r.m.s. value of the readout noises of DISC-II for dark is at present about $25 \mathrm{e}^{-}$.

\section{PHOTON STATISTICS OF CCD MICROMETER}

While the diurnal motion of star images is continuous, the pixel-to-pixel transfers of the accumulated electric charges are made regularly but in interrupted sequence. Then the mixture of the original photon intensities over two adjoining pixels along the diurnal motion is inevitable in drift-scanning method. Even in this situation an effective location estimation of image centers can be made by using a trimmed median filter. Under the circumstances that the thermal and read-out noises are negligibly small compared to the signal and night sky counts, the mean error of image location estimation by using a trimmed median filter is given by the following equation ( cf. Høg 1970 ):

$$
\sigma=\frac{\sqrt{C_{*}+C_{\mathrm{sky}}}}{2\left(C_{*} / s\right)} \approx \frac{s}{2} \times\left(\frac{S}{N}\right)^{-1},
$$


where $C_{*}$ is the total accumulated number of photoelectrons of the object, $C_{\text {sky }}$ the total sky counts evaluated over the adopted median filter, and $s$ the seeing size.

For the combination of DISC-II + Tokyo PMC at Mitaka, $C_{*} \sim 1500 \mathrm{e}^{-}$and $C_{\text {sky }} \sim$ $7500 \mathrm{e}^{-}$for 15 th mag stars at $\delta=0^{\circ}$ (integration time $=138 \mathrm{sec}$ ) when the width of the median filter is assumed to be five pixel widths both in the vertical and horizontal directions. Thus $\sigma=0.095 "(\approx 1 / 19$ of a pixel width) for $s=3$ ". This value is close to that estimated by numerical simulation (Stone 1989). Note that the mean error of the image location estimation caused by the atmospheric image fluctuations is about 0.10 " for the integration time of $138 \mathrm{sec}(\mathrm{H} \varnothing \mathrm{g} 1968)$.

\section{OBSERVATIONS WITH CCD MICROMETER}

\subsection{Optical Position of 3C273B}

The right ascensions of quasar $3 \mathrm{C} 273$ and some faint stars around the quasar are determined with DISC equipped to Gautier meridian circle of the National Astronomical Observatory, Mitaka. The observation was made by using the FK5 stars as the reference stars, and the observed position of $3 \mathrm{C} 273 \mathrm{~B}$ was reduced to the position of J2000.0 equinox and equator. It is shown that the observed R.A. of the quasar is

$$
12^{\mathrm{h}} 29^{\mathrm{m}} 06^{\mathrm{s}} 695 \text { (obs. epoch 1991.41), }
$$

which is within 0.07 arcsec from the best possible radio position (R.A.) of the quasar (cf. Réquième and Mazurier 1991).

\subsection{Observation of Nuclei of Galaxies with CCD Meridian Circle}

Some galaxies have bright compact nuclei. It is very interesting for us to know the accuracy with which we can estimate the position of the photo center of those galaxies. As a starting trial we performed with our CCD meridian circle (DISC or DISC-II + Gautier meridian circle) several observations of the Seyfert galaxy NGC 1068. From the studies of the observed projected intensities of the central part of NGC 1068, it is seen that the nucleus of NGC 1068 can be usable for the definition of the center of the galaxy. Within our limited experiments we found that by using a simple split median filter ( $c f$. Yoshizawa, et al. 1985) the photocenter can be linked to the FK5 system with the accuracy better than 0.2 " by a single observation.

\section{SECOND TOKYO PMC PROGRAM WITH CCD MICROMETER}

The first Tokyo PMC program has been observed since 1985 and will terminate in March, 1993 (Yoshizawa et al. 1991). A group of works of overhaul and modifications of the telescope system is needed for 6 to 9 months before the commencement of the second Tokyo PMC program. As is stated in the previous sections the CCD micrometer will be introduced in the second Tokyo PMC program, i.e., a part of the second program will be observed with DISC-II. Because of the characteristics of the drift-scanning method, it is not practical to observe the stars of high declinations. Thus our main targets to be observed with DISC-II must be located in the declination zones $-40^{\circ}$ to $+40^{\circ}$. At present we are proposing to accomplish the digital strip mappings of almost all regions of $-25^{\circ} \leq \delta \leq 35^{\circ}$ with two-fold overlapping duplicity. The work will require in total about 4500 hours out of 
the available observation times, and the total amount of raw data shall be about $700 \mathrm{~GB}$ if no data compressions are done.

It is not a simple task to relate the coordinates of the mapped data to the fundamental reference frame, i.e. the coordinates of the FK5 system. Thus the observations of the FK5 stars at an appropriate time interval are highly advisable during the course of the digital strip mappings, if not indispensable, to achieve the reliable links. Note that we can find 46 QSOs and galaxies brighter than 17th V-mag among the list of 234 strong compact extragalactic radio sources of Argue et al. (1984). The link of the optical reference frames to VLBI frames through those extragalactic objects is also a work worth to be performed.

The authors would like to express their sincere thanks to Prof. M. Miyamoto for his constant encouragement given to them from the beginning of the present work.

\section{REFERENCES}

Argue, A.N., de Vegt, C., Elsmore, B., Fanselow, J., Harrington, R., Hemenway, P., Johnston, K.J., Köhr, H., Kumkova, I., Niell, A.E., Walter, H., and Witzel, A. 1984, Astron. Astrophys. 130, 191.

$\mathrm{H} \varnothing \mathrm{g}$, E. 1968, Z. Astrophys. 69, 313.

$\mathrm{H} \varnothing \mathrm{g}$, E. 1970, Astron. Astrophys., 4, 89.

Monet, D.G. 1988, Ann. Rev. Astron. Astrophys., 26, 413.

Réquième, Y. and Mazurier, J.M. 1991, Astron. Astrophys. Suppl. Ser., 89, 311.

Stone, R.C. 1989, Astron. J., 97, 1227.

Stone, R.C. 1991, paper presented at XXIst G.A. of IAU, Buenos Aires.

Yoshizawa, M., Andreasen, G.K., and Høg, E. 1985, Astron. Astrophys., 147, 227.

Yoshizawa, M., Suzuki, S., and Miyamoto, M. 1991, in "Reference Systems"(IAU Coll.

No. 127)(U.S.Naval Obs., Washington, D.C.), eds. J.A.Hughes, C.A.Smith, G.H.

Kaplan, p.395. 